\title{
Axially and Helically Chiral Cationic Radical Bicarbazoles: SOMO-HOMO Level Inversion and Chirality Impact on the Sta- bility of Mono- and Diradical Cations
}

\author{
Sitthichok Kasemthaveechok, ${ }^{\dagger}$ Laura Abella, ${ }^{\ddagger}$ Marion Jean, ${ }^{\S}$ Marie Cordier, ${ }^{\dagger}$ Thierry Roisnel, ${ }^{\dagger}$ Nicolas \\ Vanthuyne, ${ }^{\S}$ Thierry Guizouarn, ${ }^{\dagger}$ Olivier Cador, ${ }^{\dagger}$ Jochen Autschbach, ${ }^{*},{ }_{\dagger}^{\ddagger}$ Jeanne Crassous, ${ }^{\dagger}$ and Ludovic \\ Favereau, $*,+$
}

${ }^{\dagger}$ Univ Rennes, CNRS, ISCR - UMR 6226, F-35000 Rennes, France.

‡ Department of Chemistry, University at Buffalo, State University of New York, Buffalo, New York 14260, USA.

$\S$ Aix Marseille University, CNRS Centrale Marseille, iSm2, 13284 Marseille, France.

\begin{abstract}
We report persistent chiral organic mono- and diradical cations based on bicarbazole molecular design with an unprecedented stability dependence on the type of chirality, namely axial versus helical. An unusual chemical stability was observed for sterically unprotected axial bicarbazole radical, in comparison with monocarbazole and helical bicarbazole ones. Such results were experimentally and theoretically investigated, revealing an inversion in energy of the singly occupied molecular orbital (SOMO) and the doubly highest occupied molecular orbital (HOMO) in both axial and helical bicarbazole monoradicals, along with a subtle difference of electronic coupling between the two carbazole units, which is modulated by their relative dihedral angle and related to the type of chirality. Such findings allowed us to explore in-depth the SOMO-HOMO inversion (SHI) in chiral radical molecular systems and provide new insights regarding its impact on the stability of organic radicals. Finally, these specific electronic properties allowed us to prepare a persistent, intrinsically chiral, diradical which notably displayed near infrared electronic circular dichroism responses up to $1100 \mathrm{~nm}$ and almost degenerate singlet-triplet ground states with weak antiferromagnetic interactions evaluated by magnetometry experiments.
\end{abstract}

\section{INTRODUCTION}

Stable organic molecules with open-shell electronic structures have attracted significant interest in chemistry and biochemistry due to their specific reactivity and their use as agents for in-vivo imaging. ${ }^{1,2,3}$ This class of compounds has also received increasing attention in materials science, because of the possibility to combine electron and spin conduction in molecular electronics such as organic light-emitting diodes (OLEDs), organic field-

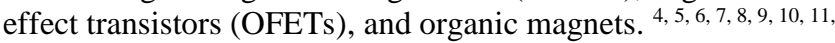
$12,13,14,15,16,17$ In this context, organic diradicals are of particular interest since they can adopt two different spin states, namely singlet and triplet, related to the interaction between the unpaired electrons within the molecular backbone. ${ }^{4,18}$ Organic $\pi$ conjugated radicals also allow to gain fundamental insights on the nature of chemical bonds and their delocalization, in addition to their other features such as narrow HOMO-LUMO energy gaps, low-lying doubly-excited states, and redox amphoterism. ${ }^{5,10,11,19}$ It has recently emerged that combining these electronic properties with a chiral organic $\pi$-conjugated system gives rise to molecular architectures with unique magnetic and chiropto-electronic features, such as specific absorption of near infrared (NIR) circularly polarized (CP) light, 20, 21, 22, 23, 24, 25, 26, 27, 28, 29, 30 radical CP luminescence (CPL), ${ }^{31,32}$ and possibly enhanced spin-filtering properties. ${ }^{33}$ However, designing organic chiral open-shell chromophores remains a formidable challenge, owing to the high chemical reactivity of radicals and poor configurational stability. ${ }^{34}$ As a result, known examples remain rare and are mainly limited to monoradicals, with very few examples of stable diradicals. ${ }^{24,25,27,28}$ So far, the chemical and configurational stabilities issues have been overcome using mainly two strategies: Shielding the unpaired electrons with bulky substituents, or enhancing their delocalization over the molecular backbone. ${ }^{8,9,27,35,36,37,38}$ While such approaches can be efficient, they often require additional synthetic steps and may preclude significant intermolecular interactions for charge and spin transport applications. ${ }^{17}$

Recently, theoretical and experimental studies have reported that the stability of radicals can be considerably enhanced if they are in an uncommon electronic configuration, such that the energy of the singly occupied molecular orbital (SOMO) is below the highest doubly occupied molecular orbital (HOMO) level (Figure 1). Systems with such a SOMO-HOMO inversion (SHI), which has sometimes (albeit imprecisely ${ }^{39}$ ) been construed as a 'non-Aufbau' electronic configuration, ${ }^{21,}$,27, 40, 41, 42 have been notably successful as achiral luminescent radical emitters in OLED devices. ${ }^{43}$ While this approach has appeared rather interesting to increase the stability of already persistent radicals such as nitroxide and tris(2,4,6-trichlorophenyl)methyl (TTM, Figure 1), the impact of SHI on known unstable radical remains unexplored. Regarding chiral radicals, a SHI has been mentioned only for one helical derivative under its racemic form. ${ }^{21}$ In addition, compounds with such a peculiar electronic configuration are often viewed as promising intermediates to form triplet ground state diradicals with nondegenerate SOMOs upon oxidation, resulting from the removal of one electron of the HOMO level. ${ }^{21,42,44}$ Accordingly, a deep understanding of the electronic and steric factors that govern monoradical stability displaying a SHI is urgently needed, as it may help to develop open-shell compounds with higher stability and bring 
new synthetic strategies to design innovative chiral organic high-spin materials.

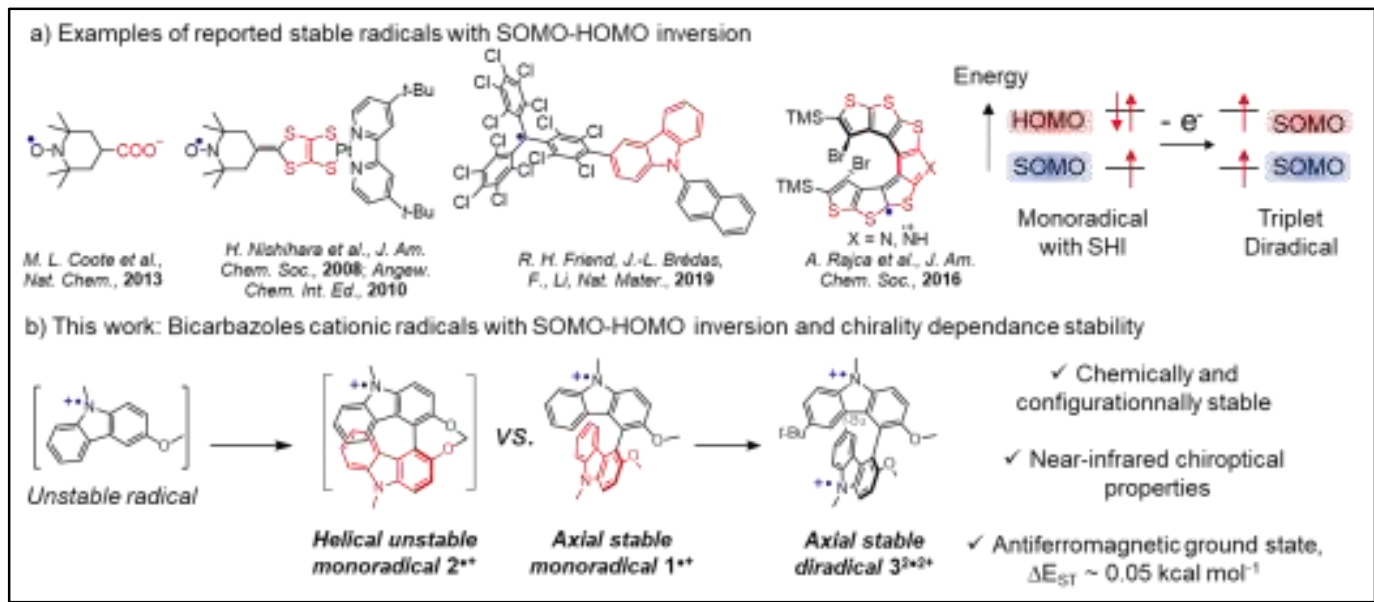

Figure 1. a) Examples of reported stable monoradicals with a SOMO-HOMO inversion (SHI) where the molecular fragment providing the HOMO level above the SOMO one is highlighted in red, and schematic illustration of triplet ground-state diradical obtained from the oxidation of a radical with a SHI ${ }^{42}$ b) Novel axially and helically mono- and diradicals cationic carbazole derivatives. The unoccupied counterpart of the SOMO in the SHI systems is calculated to be above the near-degenerate energies of the spin orbitals constituting the HOMO level, i.e., there is no electron hole among the occupied levels.

Herein, we report a simple molecular design of persistent chiral organic cationic radicals based on $C_{2}$-symmetric bicarbazole derivatives. Investigations of their photophysical, chiroptical and electrochemical properties revealed a high chemical stability for unprotected carbazole axial radical $\mathbf{1}^{\mathbf{}+}$, in comparison to unstable helical $\mathbf{2}^{\bullet+}$ (Figure 1b). Interestingly, both compounds exhibit SHI, indicating that such an electronic configuration is not a sufficient prerequisite to provide radical stability. Our results rather suggest that in addition to the SHI, the electronic coupling between the radical center and the electron rich unit on which the HOMO is centered plays a crucial role in the radical reactivity. In $\mathbf{1}^{\bullet+}$ and $\mathbf{2}^{\bullet+}$, this aspect depends on the dihedral angle between the carbazole moieties, which is governed by the axial and helical chirality of the bicarbazole systems. ${ }^{45}$ Such findings bring new insights regarding the stability of organic radicals and allowed us to further design and isolate chemically and configurationally stable chiral diradical $\mathbf{3}^{\mathbf{2} \mathbf{2}_{+}}$, which displayed promising near-infrared chiroptical properties and nearly degenerate singlet-triplet ground states.

\section{RESULTS AND DISCUSSION}

Synthesis and Structural characterization. We first prepared bicarbazole derivatives $\mathbf{1}$ and $\mathbf{2}$. The synthesis was inspired from that of BICOL, a ligand previously used in asymmetric catalysis. ${ }^{46,47}$ Briefly, carbazole CBzOMe was first hydrolyzed and then engaged in an oxidative homocoupling reaction using vanadium complex as catalyst to afford 4,4'-bicarbazole-3,3'-diol, BICOL, derivative (Scheme 1). The latter was then alkylated both at the nitrogen and oxygen positions to give rac-1, which was resolved to its corresponding axially chiral enantiomers (+)-/(-)-1 using chiral HPLC (see ESI for details). Helical (+)-/(-)-2 were successively obtained from (+)- and (-)1 through hydrolysis and subsequent bridging of the hydroxy groups using diiodomethane in $90 \%$ yield. Further functionalization of monocarbazole CBzOMe afforded $N$-methylated 1', which was then converted to sterically hindered 3' with $t$-Butyl group at the 6-position (Scheme 1). Steric protection was also applied to (+)-/(-)-1 and gave (+)-/(-)-3, in $90 \%$ yield. Under similar conditions, helical bicarbazole 2 did not afford the expected bis $t$-Butyl derivative $\mathbf{4}$ but rather a complex mixture of oligomers with multiple $t$-Butyl groups. As a result, compound $\mathbf{4}$ was obtained from $\mathbf{3}$ using similar synthetic strategy than for $\mathbf{2}$, in an overall $25 \%$ yield.

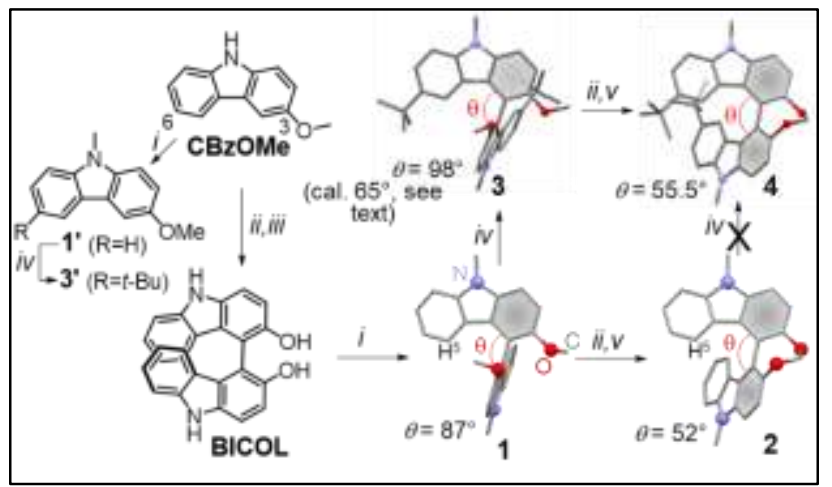

Scheme 1. Schematic synthetic route for 1, 1', 2, 3, 3' and 4. Reaction conditions: i) $\mathrm{Me}_{2} \mathrm{SO}_{4}, 22 \mathrm{M} \mathrm{NaOH}$, Acetone, reflux, $77 \%$; ii) $\mathrm{BBr}_{3}, \mathrm{CH}_{2} \mathrm{Cl}_{2}, \mathrm{rt},>95 \%$; iii) $\mathrm{VO}(\mathrm{acac})_{2}, \mathrm{MeCN}, \mathrm{O}_{2}, \mathrm{rt}$, $50 \%$; iv) $t$ - $\mathrm{BuCl}, \mathrm{ZnCl}_{2}, \mathrm{MeNO}_{2}, \mathrm{rt},>90 \%$; v) $\mathrm{CH}_{2} \mathrm{I}_{2}, \mathrm{~K}_{2} \mathrm{CO}_{3}$, acetone, Ar, reflux, $>90 \%$. ORTEP drawing of 1, 2, 3 and 4 with the corresponding dihedral angle between the carbazole units. Hydrogen atoms are omitted for clarity.

$\mathrm{X}$-Ray structures analyses provide further structural insights for rac-1 and rac-2, which crystallized in the $P-1$ and $C 2 / c$ space groups, respectively. Dihedral angles of $87.1^{\circ}$ and $52.4^{\circ}$ between the carbazole units were measured for $\mathbf{1}$ and $\mathbf{2}$, respectively, which for the latter appeared to be reminiscent of the one found for carbo[6]helicene. ${ }^{48,49,50}$ This structural difference was confirmed by density functional theory (DFT) calculations (Figure S49), and also probed by the ${ }^{1} \mathrm{H}$ NMR spectra. Protons $\mathrm{H}^{5}$ (Scheme 1, doublet signal in Figure S33, S37) are more shielded for 1 than for 2 (6.53 and $6.95 \mathrm{ppm}$, respectively), owing to an unequal current ring effect coming from either the opposite aromatic phenyl ring in $\mathbf{1}$ or the $N$-heterocycle one in $\mathbf{2}$. $R a c-3$ and $r a c-4$ crystallized in the $P 2_{1} / n$ space group and dis- 
played dihedral angles between 98 and $102^{\circ}$ for $\mathrm{rac}-3$ (two molecules within the same unit cell) and of $55.5^{\circ}$ for $\mathrm{rac}-4$. However, two conformers were found for $\mathbf{3}$ in the calculations, with dihedral angles of $65^{\circ}$ and $106.6^{\circ}$, the latter being higher in energy by $1.8 \mathrm{kcal} \cdot \mathrm{mol}^{-1}$ (Boltzmann population of $4 \%$ at $298 \mathrm{~K}$ ). Therefore, due to crystal packing, 3 does not crystallize in its lowest-energy conformer. The calculated properties of $\mathbf{3}$ further support the higher abundance of the conformer with dihedral angle of $65^{\circ}$ in solution by comparison with the experimental chiroptical properties (vide infra). All these observations indicate that the electronic coupling between the two carbazole units of these chiral compounds should differ significantly, depending on the dihedral angle, which may impact their corresponding photophysical, chiroptical and electronic properties.

Computational Details. Kohn-Sham Density functional theory (DFT) as implemented in the Gaussian (G16) package was used for all computations, ${ }^{51}$ employing the PBE0 functional $^{52}$ and the def2-SV(P) basis. ${ }^{53,54}$ For neutral and oxidized species, solvent effects were considered by means of the polarizable continuum model (PCM) for dichloromethane and acetonitrile, ${ }^{55}$ respectively, to match the experimental conditions. 'D3' dispersion corrections were included in the calculations. ${ }^{56}$ Excited state energies, transition moments, excited state structures and their vibrational normal modes were obtained from time-dependent DFT (TD-DFT) response theory. For the absorption and electronic circular dichroism (ECD) spectra we calculated the 200 lowest-energy vertical spin-allowed electronic excitations. The transitions were subsequently Gaussianbroadened with $\sigma=0.20 \mathrm{eV}$ to simulate the spectral envelopes. For overviews of the theoretical approach to model natural optical activity by quantum chemical calculations, in particular via TD-DFT, see, for example, available reviews. ${ }^{57,58}$ Different functionals and solvent effects were tested for these compounds; see the Supporting Information for details.

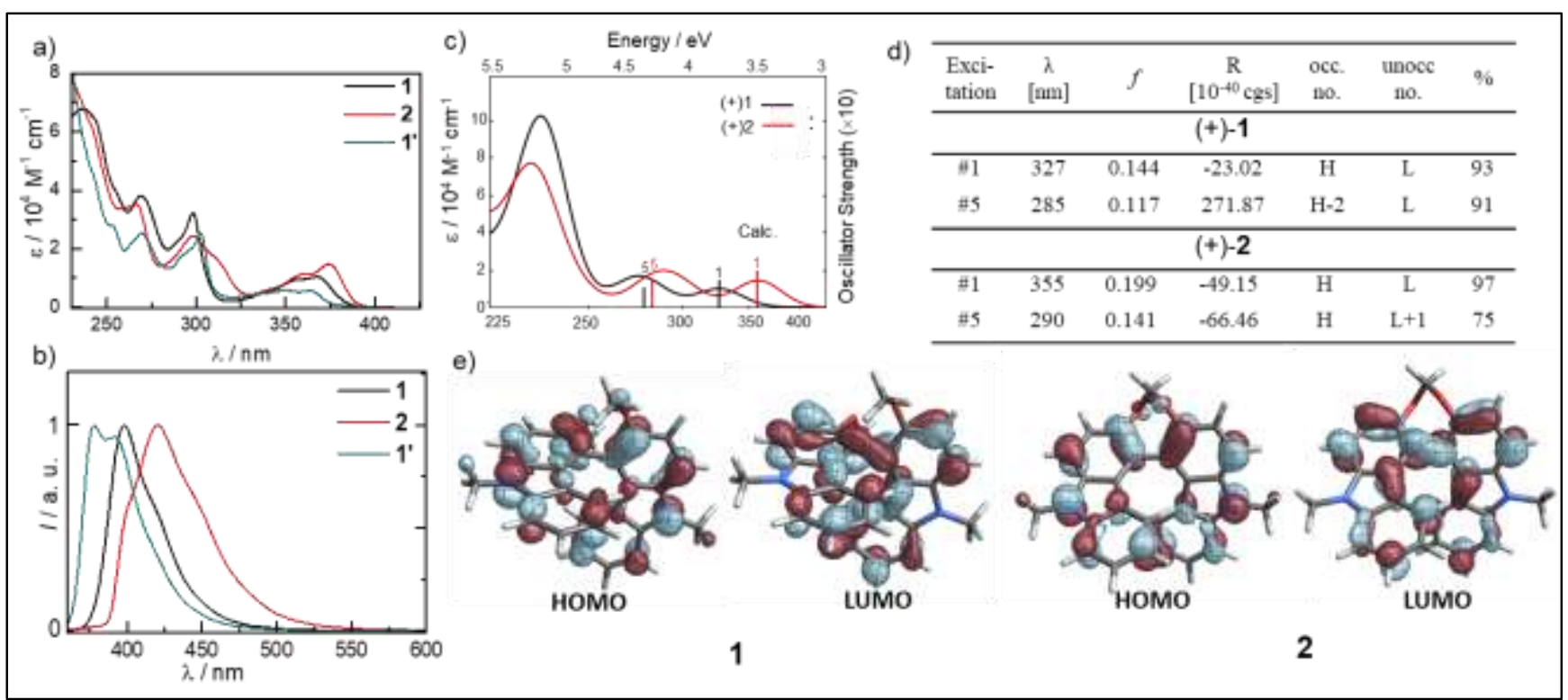

Figure 2. a) UV-vis absorption spectra of 1 (black), 2 (red) and 1' (green); b) Normalized fluorescence spectra of 1 (black), 2 (red) and 1' (green); c) Calculated (Calc.) absorption spectra for $\mathbf{1}$ (black) and $\mathbf{2}$ (red) with selected transitions and oscillator strengths indicated by 'sticks' bars and; d) Details for selected transitions and occupied (occ)-unoccupied (unocc) MO pair contributions (greater than 10\%) to the transition density for (+)-1 and (+)-2. H and L indicate the HOMO and LUMO, respectively, for which isosurfaces $( \pm 0.035 \mathrm{au})$ are shown in e).

Photophysical properties of 1, 2 and 1'. Carbazole 1' and bicarbazoles $\mathbf{1}$ and $\mathbf{2}$ display similar UV-vis spectra, with two maxima of absorption at 270 and $300 \mathrm{~nm}\left(\varepsilon \sim 3.7 \times 10^{4}\right.$ and $\left.3.0 \times 10^{4} \mathrm{M}^{-1} \mathrm{~cm}^{-1}\right)$, respectively, and a vibronic band between 325 and $390 \mathrm{~nm}\left(\varepsilon \sim 1.0 \times 10^{4} \mathrm{M}^{-1} \mathrm{~cm}^{-1}\right.$, Figure 2$)$. Whereas 1 and 2 show the expected higher absorption intensity, about twice as for 1', the UV-vis spectrum of $\mathbf{2}$ exhibits red-shifted absorption bands at 330 and $370 \mathrm{~nm}$, indicating a stronger electronic interaction between the bridged carbazole systems, as expected from the smaller dihedral angle. For both bicarbazole compounds, the lowest-energy excitation with sizeable oscillator strength $(f)$ corresponds to the HOMO-LUMO transition at 327 and $355 \mathrm{~nm}$ for $\mathbf{1}$ and $\mathbf{2}$ respectively. In each case, the HOMO and LUMO are delocalized over the entire molecule, and the LUMO exhibits nodes at the nitrogens (Figure 2). Both compounds have quasi-degenerate HOMO and HOMO-1 levels, separated by $0.112 \mathrm{eV}$ and $0.149 \mathrm{eV}$ for 1 and 2, respectively. Visual inspection of the MO isosurface plots reveals that the HOMO-1 and HOMO are essentially in-phase and out-ofphase (+/-) linear combinations of individual carbazole fragment frontier orbitals (FFOs). The different energetic splitting of the HOMO-1 and HOMO indicates that the FFO interactions are more pronounced in compound $\mathbf{2}$, which goes along with the significant red-shift and slightly larger intensities for the calculated transitions at $285-290 \mathrm{~nm}$ and $327-355 \mathrm{~nm}$ in comparison to 1 (Figure 2 and Tables S7-S8). The chiroptical properties of $\mathbf{1}$ and $\mathbf{2}$ have also been studied, both experimentally and theoretically, and are detailed in the supporting information (Figure S5 and S54).

Bicarbazoles $\mathbf{1}$ and $\mathbf{2}$ display moderately intense fluorescence around $400 \mathrm{~nm}$, with quantum yields of $\sim 20 \%$ (Figure 2). The 
enhanced electronic communication between the carbazole units in $\mathbf{2}$ remains also present in the excited state as indicated by the fact that its emission peak maximum is red-shifted relative to the emission peak of $\mathbf{1}$, similar to how the absorption of $\mathbf{2}$ is red-shifted. In fact, $\mathbf{1}$ displays an emission profile close to the one of 1', evidencing a weak electronic coupling between the carbazole $\pi$-systems also in the excited-state.

Radical $\mathbf{1}^{\bullet+}$ and $\mathbf{2}^{\bullet+}$. Cyclic voltammetry $(\mathrm{CV})$ revealed two irreversible oxidation processes for $\mathbf{1}$ ' around $0.90 \mathrm{~V}$ and $1.70 \mathrm{~V}$, and two distinct oxidation events for both $\mathbf{1}$ and $\mathbf{2}$ in the range of 0.8 to $1.4 \mathrm{~V}$ (vs. SCE, Figure 3). Compound 1 exhibits a first fully reversible one-oxidation process at $0.92 \mathrm{~V}$ and a second less reversible one at $1.11 \mathrm{~V}$ with the appearance of a small reduction peak at $0.70 \mathrm{~V}$ in the reverse scan. In stark contrast, 2 showed two highly irreversible oxidation processes at +1.14 and $+1.40 \mathrm{~V}$, as classically observed for 3- and/or 6-unprotected carbazole derivatives like 1', which form unstable cations under oxidative conditions. ${ }^{59,60,61}$ The unusual reversibility of the first oxidation event of $\mathbf{1}$, as well as the marked difference of $\sim 200 \mathrm{mV}$ with the one of $\mathbf{2}$, prompted us to deeper investigate monoradical cation $\mathbf{1}^{\bullet+}$. For comparison purpose, 3 and 6-protected monocarbazole 3' was selected as a persistent carbazole radical reference since its $\mathrm{CV}$ gives one fully reversible oxidation process at $0.87 \mathrm{~V} v s$. SCE (Figure 3).

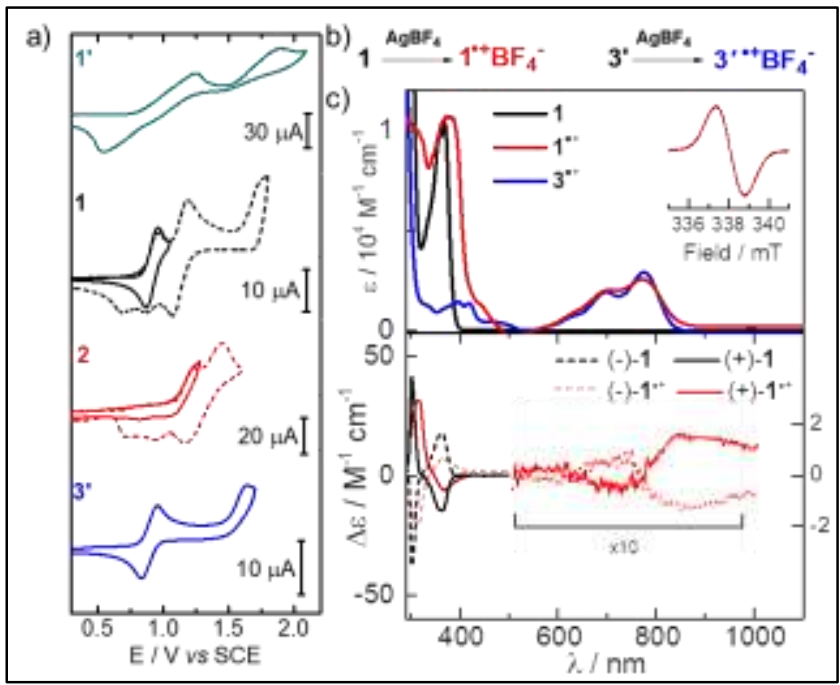

Figure 3. a) Cyclic voltammograms of 1' (green), 1 (black), 2 (red) and 3' (blue) versus saturated calomel electrode (SCE); b) Synthetic route to persistent radical $\mathbf{1}^{\bullet+} \mathbf{B F}_{4}^{-}$(red) and $3^{\bullet \bullet+}$ $\mathbf{B F}_{4}^{-}$(blue); c) Top: UV-vis-NIR absorption spectra of $\mathbf{1}$ (black), $\mathbf{1}^{\bullet+} \mathbf{B F}_{4}^{-}$(red) and $3^{{ }^{\bullet \bullet+}} \mathbf{B F}_{4}^{-}$(blue) in dichloromethane at $298 \mathrm{~K}$, inset: X-band EPR $(v=9.4858 \mathrm{GHz})$ of $\mathbf{1}^{\mathbf{}} \mathbf{B F}_{\mathbf{4}}^{-}$; bottom :ECD spectra of (+)-1 (black) and (+)-1 ${ }^{\bullet+} \mathbf{B F}_{4}^{-}$(red) with their corresponding enantiomers in dash line in dichloromethane at $298 \mathrm{~K}$ with an extension of the NIR-ECD spectra of (+)- and (-)-1 $\mathbf{1}^{\bullet+}$ $\mathrm{BF}_{4}$.

Addition of $\mathrm{AgBF}_{4}$ as a chemical oxidant to a solution of $\mathbf{3}^{\prime}$ afforded persistent radical $3^{{ }^{\bullet}+} \mathbf{B F}_{4}^{-}$, as expected for 3,6-protected carbazole, with a characteristic vibronic visible signature at 696 $\mathrm{nm}$ and $771 \mathrm{~nm}$ (Figure 3). ${ }^{59,60,62}$ Using the same conditions, 1 was quantitatively converted to $\mathbf{1}^{{ }^{+}} \mathbf{B F}_{4}^{-}$, which showed similar

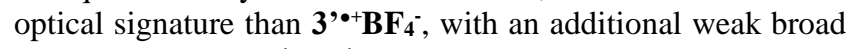
IR band $\left(\varepsilon \sim 500 \mathrm{M}^{-1} \mathrm{~cm}^{-1}\right.$ at $\left.1000 \mathrm{~nm}\right)$, and a half-life evaluated to 3 hours in air saturated $\mathrm{CH}_{2} \mathrm{Cl}_{2}$. Under inert conditions, this half-life extends to 14 hours, indicating a persistent character for $\mathbf{1}^{\bullet+} \mathbf{B F}_{4}$. Indeed, mass spectroscopy and $\mathrm{UV}$-vis analysis of the resulting mixture after decomposition of the radical compound reveal the presence of bicarbazole oligomers of different molecular size, which further confirm the kinetic stability of $\mathbf{1}^{\bullet+} \mathbf{B F}_{4}{ }^{-}$. The fact that the half-life of the radical decreases in presence of oxygen indicates that other decomposition pathways may also take place.

The paramagnetic nature of these two monoradical cations was also assessed by EPR measurements, which display a similar intense doublet signal of an organic nitrogen based radical for both compounds ( $g=2.00$, Figure 3 and Figure S16). The identical optical and magnetic signatures of $3^{{ }^{\bullet \bullet}+} \mathbf{B F}_{4}{ }^{-}$and $\mathbf{1}^{\bullet+} \mathbf{B F}_{4}$, and the lack of intense intervalence absorption transition for the latter, suggest that the unpaired electron is localized mainly on one carbazole unit in $\mathbf{1}^{{ }^{\bullet+}} \mathbf{B F}_{4}^{-}$, and only slightly delocalized over the second one, contrary to class II and III of organic mixed valence compounds. ${ }^{63}$ This situation is rather surprising since it leads to an unprecedented persistent carbazole centered radical without any steric protection at the reactive 6-position.

According to the photophysical experiments and theoretical calculations (Figure 2), the two carbazole fragment orbitals in $\mathbf{1}$ showed negligible interaction due to their relative nearly perpendicular orientation, affording almost degenerate HOMO and HOMO-1 levels in the neutral state. As a result, the first two oxidation events occurring for $\mathbf{1}$ are the sequential oxidations of each carbazole unit, which partially lift the pseudo-degeneracy of the HOMO in the monoradical cationic state $\mathbf{1}^{\mathbf{}}$. . These two anodic signals are followed by a third irreversible one at +1.63 $\mathrm{V}$, attributed to the second oxidation of one $\mathrm{CBz}^{\bullet+}$ unit, in accordance with the sequential oxidations observed for monocarbazole $3^{\prime}$ at $+0.85\left(\mathbf{3}^{, \bullet+}\right)$ and $+1.53 \mathrm{~V}\left(\mathbf{3}^{, \mathbf{2} \bullet 2+}\right.$, Figure 3$)$. Following these considerations, $\mathbf{1}^{\mathbf{}+} \mathbf{B F}_{4}{ }^{-}$adopts an uncommon electronic configuration since its SOMO becomes lower in energy than the remaining HOMO of the second non-oxidized carbazole fragment, partially isolated from the radical center due to the high dihedral angle between the two $\pi$-systems.

Further insights regarding the SHI were obtained by theoretical analysis of the electronic configuration of $\mathbf{1}^{\mathbf{}+} \mathbf{B F}_{4}^{-}$. As depicted in Figure 4, the calculated spin densities are represented by spin-polarized Kohn-Sham (KS) systems where the $\alpha$-spin HOMO has a matching occupied $\beta$-spin HOMO, with both having very similar orbital energies, while the SOMO lies energetically below the HOMO-1. Unlike the HOMO and HOMO-1 on the neutral systems, the frontier orbitals of $\mathbf{1}^{\mathbf{}}$ are localized on either one of the fragments. The calculated spin density of $\mathbf{1}^{\bullet+}$ clearly reflects the excess $\alpha$-spin represented by the SOMO and the corresponding $\beta$-spin hole represented by the LUMO. As mentioned already, this SHI has been claimed as a stabilizing strategy for radicals, ${ }^{21,40,41,42,43,64}$ and our results suggest that such effects may be also responsible for the observed stability of $\mathbf{1}^{\bullet+} \mathbf{B F}_{4}^{-}$.

It is important to note, however, that $\mathbf{2}^{\bullet+} \mathbf{B F}_{4}{ }^{-}$appeared experimentally unstable, despite an electronic configuration implying a SHI, and instantaneously affords a complex mixture of oligomers. This high stability contrast represents therefore a unique opportunity to gain more insights about the key parameters that lead to stable radical with SHI configuration. We note that different functionals have been tested to ensure that the SHI for $\mathbf{1}^{\bullet+}$ and $\mathbf{2}^{\bullet+}$ does not only show up with one specific functional (see SI). Since the structure of $\mathbf{1}$ and $\mathbf{2}$ differ by the dihedral angle between the two carbazole systems, the difference of reactivity between $\mathbf{1}^{\bullet+} \mathbf{B F}_{4}^{-}$and $\mathbf{2}^{\bullet+} \mathbf{B F}_{4}^{-}$may be related either to steric arguments or to the electronic coupling between the radical center 
and the second carbazole fragment. As mentioned above, both radicals $\mathbf{1}^{\bullet+} \mathbf{B F}_{4}{ }^{-}$and $\mathbf{2}^{\bullet+} \mathbf{B F}_{4}{ }^{-}$show a similar decomposition pathway implying the formation of oligomers occurring presumably at the remaining free 6 and 6' positions, as it occurs for classical non protected carbazole radicals. Since the second carbazole unit is not attached next to this reactive position of the carbazole radical unit, and less well oriented for $\mathbf{1}^{\bullet+} \mathbf{B F}_{4}{ }^{-}$than for $\mathbf{2}^{\bullet+} \mathbf{B F}_{4}$ (related to their dihedral angles, Figure S49), the substituent steric hindrance seems too far to play a decisive role in the difference of stability between $\mathbf{1}^{\bullet+} \mathbf{B F}_{4}^{-}$and $\mathbf{2}^{\bullet+} \mathbf{B F}_{4}{ }^{-}$. On the contrary, the electronic properties between $\mathbf{1}$ and $\mathbf{2}$ clearly differ, as evidence by the photophysical and chiroptical characterizations, which show that the electronic interaction between the two carbazole units is negligible for $\mathbf{1}$ in comparison to $\mathbf{2}$. This is fur- ther supported by the separation of the two first oxidation potentials, $\Delta E_{\mathrm{Ox}}$, equal to $190 \mathrm{mV}$ and $260 \mathrm{mV}$ for $\mathbf{1}$ and $\mathbf{2}$, respectively (Figures 3 and 4). ${ }^{63,65}$ In addition, the second carbazole unit does not help to stabilize the radical by resonance effect due to its meta-position regarding the nitrogen atom of the radical unit. On the contrary, it induces a negative inductive effect which destabilizes more radical $\mathbf{2}^{\bullet+} \mathbf{B F}_{4}{ }^{-}$than $\mathbf{1}^{\bullet+} \mathbf{B F}_{4}^{-}$, due to the greater electronic communication between the carbazole systems in $\mathbf{2}^{\mathbf{}} \mathbf{B F}_{\mathbf{4}}$. This is experimentally confirmed by the facility of $\mathbf{1}$ to form the corresponding monoradical $\mathbf{1}^{\bullet+}\left(E_{\text {Ox }}=0.92 \mathrm{~V}\right.$ $v s$. SCE) as opposed to $\mathbf{2}^{\bullet+}\left(E_{\mathrm{Ox}}=1.14 \mathrm{~V} v s\right.$. SCE, Figure 4), and theoretically reproduced by the corresponding calculated vertical ionization potentials, which indicate a higher value for $\mathbf{2}(5.42 \mathrm{eV})$, in comparison to $\mathbf{1}(5.16 \mathrm{eV}$, Figure 4$)$. The difference amounts to $25 \mathrm{~kJ} / \mathrm{mol}$.

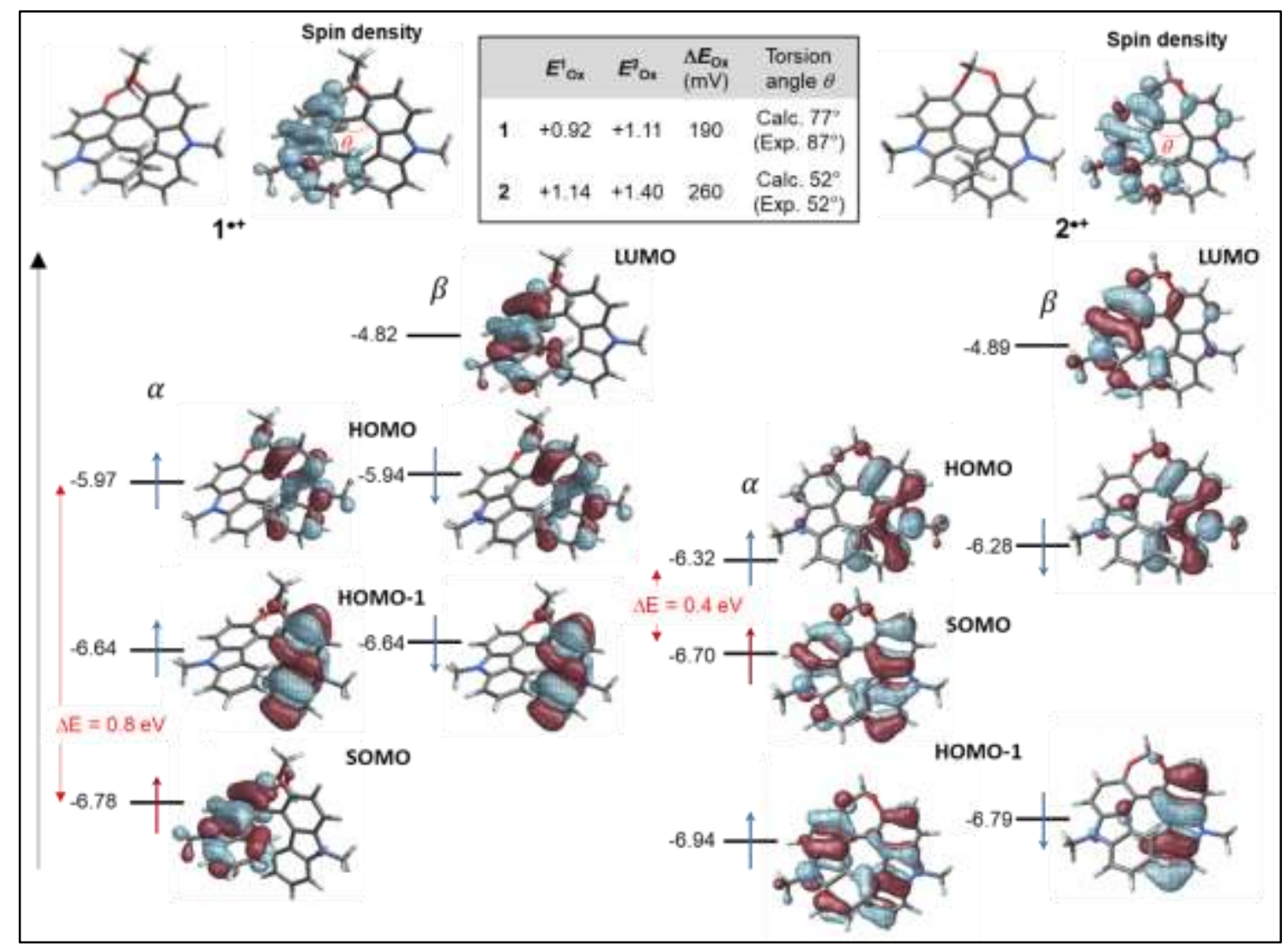

Figure 4. Calculated spin density with an isosurface of $\pm 0.002 \mathrm{au}$, orbital energies (in eV) and isosurfaces $( \pm 0.030)$ of frontier molecular orbitals computed for monoradicals $\mathbf{1}^{\bullet+}$ (left) and $\mathbf{2}^{\bullet+}$ (right). The HOMO-SOMO energy differences are averaged over the highest occupied spin orbital energies. Oxidation potentials and separation of the two first oxidation potentials in V vs. SCE, theoretical (calc.) and experimental (exp.) dihedral angles between the carbazole fragments.

Another notable difference between $\mathbf{1}^{\bullet+}$ and $\mathbf{2}^{\bullet+}$, is the orbital energy gap $\Delta E$ between the HOMO and the SOMO of $0.8 \mathrm{eV}$ and $0.4 \mathrm{eV}$, respectively. Moreover, the energy difference between the HOMO of $\mathbf{2}$ and $\mathbf{2}^{\bullet+}$ is $0.54 \mathrm{eV}$, much larger than the difference of $0.18 \mathrm{eV}$ for $\mathbf{1}$ and $\mathbf{1}^{\bullet+}$, confirming the stronger interaction between the radical center and the second carbazole in $\mathbf{2}^{\bullet+}$ (Figures 4, S57 and S58). The obtained results bring further evidence of the crucial role of the electronic coupling between the radical center and the neutral electron-rich fragment on stabilizing a molecular open-shell electronic state with SOMOHOMO energy inversion. ${ }^{43}$

In addition to its chemical stability, $\mathbf{1}^{\bullet+} \mathbf{B F}_{4}^{-}$remains also configurationally stable, presumably because of the high enantiomerization barrier of its corresponding neutral form $\left(\Delta \mathrm{G}^{\neq}=\right.$
$173.5 \mathrm{~kJ} \mathrm{~mol}^{-1}, 180^{\circ} \mathrm{C}$ in 1,2-dichlorobenzene), which allowed to record the ECD of the radical enantiomers (+)-/ $(-)-\mathbf{1}^{\bullet+} \mathbf{B F}_{4}^{-}$. As depicted in Figure 3, the ECD signals of the neutral form experienced a decrease in intensity and a global red shift of around $10 \mathrm{~nm}$ in the radical state, together with the appearance of new ECD active bands across the visible and the IR regions. For instance, the intense signal at $302 \mathrm{~nm}(\Delta \varepsilon=+40$ $\left.\mathrm{M}^{-1} \mathrm{~cm}^{-1}\right)$ and $360 \mathrm{~nm}\left(\Delta \varepsilon=-14 \mathrm{M}^{-1} \mathrm{~cm}^{-1}\right)$ for neutral (+)-1 decreased and broadened to 312 and $370 \mathrm{~nm}(\Delta \varepsilon=+24$ and $4.5 \mathrm{M}^{-1} \mathrm{~cm}^{-1}$, respectively) upon formation of $(+)-\mathbf{1}^{\bullet+} \mathbf{B F}_{4}$, all these changes being well reproduced by calculated ECD spectra (Figure S60). Importantly, new mirror-image Cotton effects at 570,715 and $860 \mathrm{~nm}\left(\Delta \varepsilon=+0.3,-0.6\right.$ and $+1.6 \mathrm{M}^{-1} \mathrm{~cm}^{-1}$, 
respectively) can be unambiguously associated to radical absorption transitions, highlighting the intrinsically chiral nature of this new persistent radical.

\section{Mono and diradical of 3 and 4 and related char-} acterization. The SHI found for $\mathbf{1}^{\bullet+} \mathbf{B F}_{4}{ }^{-}$gives an interesting opportunity to generate the corresponding organic diradical. As illustrated in Figure 1, oxidation of monoradical displaying a SHI may result in a diradical with triplet ground state multiplicity, in accordance with Hund's first rule, or an open-shell singlet with antiferromagnetic coupling of the unpaired spins (Figure S20). In addition, as $\mathbf{1}^{\mathbf{}+} \mathbf{B F}_{\mathbf{4}}$ can be considered as an innovative molecular design of chiral organic spin-polarized donor, ${ }^{16,66,67}$, $68,69,70$ it appears interesting to investigate the magnetic interaction between the two radical units upon oxidation of the second carbazole unit. In order to evaluate the possibility to obtain stable chiral diradicals, oxidation of $\mathbf{1}^{\bullet+}$ was investigated by using tris(4-bromophenyl)aminium hexachloridoantimonate ("magic blue" oxidant, $\left.(4-\mathrm{BrPh})_{3} \mathrm{NSbCl}_{6}\right) .{ }^{71}$ However, UV-vis titration indicated instantaneous reactivity of $\mathbf{1}^{\mathbf{2} \cdot 2+} \mathbf{2} \mathbf{S b F}_{\mathbf{6}}$, presumably through polymerization reaction as indicated by the formation of a broad IR band between 800 and $1800 \mathrm{~nm}$ (Figure S18) and anticipated by the non-fully reversible second oxidation process of $\mathbf{1}$ for in CV (Figure 3). To circumvent this aspect and increase radical persistence, we turned our attention to $\mathbf{3}$ and $\mathbf{4}$, which are the 6 and 6' sterically protected analogues of $\mathbf{1}$ and $\mathbf{2}$. These new chiral systems show similar chiroptical and photophysical properties as $\mathbf{1}$ and $\mathbf{2}$, but slightly red-shifted, likely owing to the presence of the donor $t$-butyl groups (Figure S5, S6). Regarding the possible presence of two conformers of $\mathbf{3}$ with different dihedral angles, the calculated ECD spectra of the conformer with the small dihedral angle $\left(67^{\circ}\right)$ agree well with the experimental ones (Figure S61), confirming its higher abundance in solution over the conformer with the large dihedral angle $\left(106^{\circ}\right)$. As for $\mathbf{1}$ and 2, CVs of $\mathbf{3}$ and $\mathbf{4}$ afford two oxidation events for both compounds (Figure 5), suggesting a SHI for corresponding monoradicals $3^{\bullet+}$ and $\mathbf{4}^{\bullet+}$, as in the case of $\mathbf{1}^{\bullet+}$ and $\mathbf{2}^{\bullet+}$. Interestingly, the reversibility of the oxidations processes was strongly enhanced in comparison to the ones for $\mathbf{1}^{\mathbf{}+}$ and $\mathbf{2}^{\bullet+}$, highlighting the benefits of the steric protection on the carbazoles 6 and 6' positions. Indeed, 3 shows full reversibility for both the first and second oxidation signals $\left(\mathrm{E}_{\mathrm{Ox}}=0.87\right.$ and 1.10 $\mathrm{V} v s$. SCE) with no degradation after cycling scans. In contrast, while the first oxidation process of $\mathbf{4}$ exhibits a fully reversible response at $1.06 \mathrm{~V} v s$. SCE, its second oxidation at $1.37 \mathrm{~V}$ shows only a partial reversibility and leads to the appearance of a weak new reduction peak at $0.85 \mathrm{~V}$ in the reverse scan, suggesting some instability for diradical $\mathbf{4}^{\mathbf{2} \mathbf{2 +}}$, as in the case of $\mathbf{1}$.

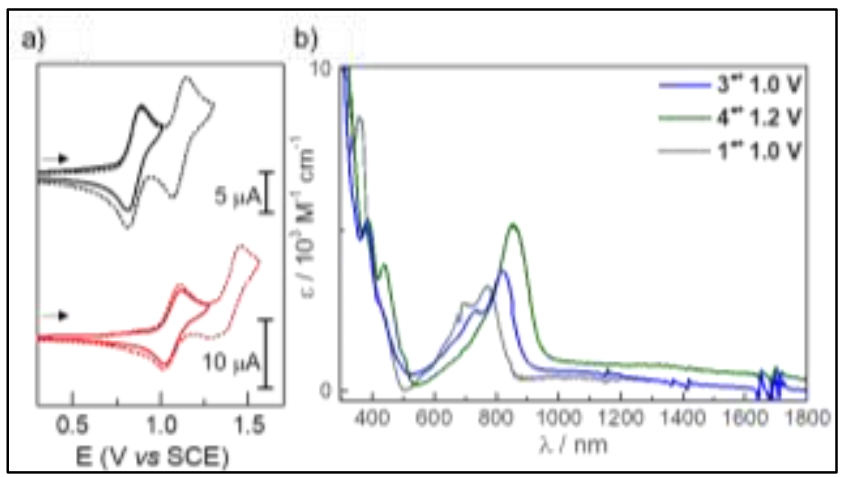

Figure 5. a) Cyclic voltammograms of $\mathbf{3}$ (black) and $\mathbf{4}$ (red) versus SCE; b) UV-vis-NIR absorption spectra of $\mathbf{1}^{\bullet+}$ (grey), $3^{\bullet+}$ (blue) and $\mathbf{4}^{\bullet+}$ (green) recorded by spectroelectrochemistry (see ESI for details).

The UV-vis-NIR spectra of monoradicals $3^{\bullet+}$ and $\mathbf{4}^{\bullet+}$ were investigated using spectroelectrochemistry and show similar pho-

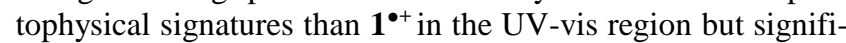
cant changes in the low energy part of the spectra (Figure 4). For both $\mathbf{3}^{\bullet+}$ and $\mathbf{4}^{\bullet++}$, the corresponding NIR absorption bands red-shift to 812 and $850 \mathrm{~nm}$ respectively, and show much less vibronic structure than $\mathbf{1}^{\circ+}$. In addition, the broad NIR band that extends up to $1800 \mathrm{~nm}$ for both radicals, appears more intense than for $\mathbf{1}^{\bullet+}$, with $\varepsilon=760 \mathrm{M}^{-1} \mathrm{~cm}^{-1}$ and $1100 \mathrm{M}^{-1} \mathrm{~cm}^{-1}$ for $3^{\bullet+}$ and $\mathbf{4}^{\bullet+}$, respectively. These observed optical differences suggest that the radical center is not anymore localized on one carbazole fragment for both $3^{\bullet+}$ and $4^{\bullet+}$ but interacts with the second donor fragment to some extent. Moreover, the electronic coupling between the two carbazole seems higher for helical radical $4^{\bullet+}$ $\left(\Delta E_{\mathrm{Ox}}=310 \mathrm{mV}\right)$ than for axial $3^{\circ+}\left(\Delta E_{\mathrm{Ox}}=230 \mathrm{mV}\right)$, in line with its smaller dihedral angle $\left(54^{\circ}\right.$ vs. $65^{\circ}$ for $4^{\bullet+}$ and $3^{\bullet+}$, respectively). Despite the presence of the $t$-butyl groups, addition of one equivalent of magic blue to an acetonitrile solution of $\mathbf{4}$ did not lead to $\mathbf{4}^{\bullet+} \mathbf{S b C l}_{\mathbf{6}}$, but rather polymeric materials as indicated by the obtained broad NIR band centered at $1440 \mathrm{~nm}$ which clearly differs from the optical signature recorded by spectroelectrochemistry (Figure S10). This result further indicates that the steric protection at the 6 and 6' positions of the investigated bicarbazole systems is not a decisive factor for reaching radical stability. On the contrary, $\mathbf{3}^{\bullet+} \mathbf{B F}_{4}^{-}$was quantitatively obtained and displays a high stability with a measured half-life of 4.1 days in solution (Figure S28, Table S6). For comparison, $3^{{ }^{\bullet+}} \mathbf{B F}_{4}^{-}$shows a half-life of 3.5 days under the same conditions, highlighting a synergetic effect between the steric protection and the SHI on the radical stability. Frontier molecular orbitals of $\mathbf{3}^{\bullet+}$ further show that the SHI goes along with a (HOMO/SOMO) energy gap of $0.47 \mathrm{eV}$, which is in between the ones for $\mathbf{1}^{\bullet+}$ and $\mathbf{2}^{\mathbf{}+}$. This follows the trend for the calculated dihedral angles between the $\pi$-conjugate systems $\left(77^{\circ}\right.$ and $52^{\circ}$ for $\mathbf{1}^{\bullet+}$ and $2^{\bullet+}$, respectively). These results further confirm the importance of minimizing the electronic interaction between the radical center and the additional donor unit to increase stability of a radical displaying SHI. The high stability found for $\mathbf{3}^{-+} \mathbf{S b C l}_{6}$ prompted us to further explore the formation of diradical $\mathbf{3}^{\mathbf{2} \mathbf{2}+} \mathbf{2 S b C l}_{\mathbf{6}}$. Additions of two equivalents of magic blue to the solution of 3 results in the quantitative formation of diradical dication $\mathbf{3}^{\mathbf{2} \mathbf{2}+} \mathbf{2} \mathbf{S b C l}_{\mathbf{6}}$, which represents one of the rare examples of persistent intrinsically organic chiral diradicals, and thus embedded unique magnetic and chiroptical properties (Figure 6). $\mathbf{3}^{\mathbf{2} \boldsymbol{2}+} \mathbf{2} \mathbf{S b C l}_{6}^{-}$displayed similar UV-visNIR signature than $\mathbf{3}^{\bullet+} \mathbf{S b C l}_{6}^{-}$, with twice higher absorption intensity, as expected from the presence of two oxidized carbazole units (Figure 6). The UV-vis-NIR spectrum of $\mathbf{3}^{\mathbf{2 \bullet}+} \mathbf{2 S b C l}_{\mathbf{6}}{ }^{-}$ shows a $20 \mathrm{~nm}$ blue shift of the signal at $800 \mathrm{~nm}$ in comparison to $\mathbf{3}^{-+} \mathbf{S b C l}_{\mathbf{6}}$, along with an increase and red-shift of the broad IR band $\left(\varepsilon=2250 \mathrm{M}^{-1} \mathrm{~cm}^{-1}\right.$ at $\left.1400 \mathrm{~nm}\right)$, suggesting some interactions between the radical centers. The calculated spectrum of $3^{2 \bullet 2+}$ reproduced well the experimental one and notably allowed us to assign the low-energy bands between 700 and $1200 \mathrm{~nm}$ to transitions among the set HOMO-1, HOMO and LUMO, LUMO+1 (Figure S66 and Table S14). 


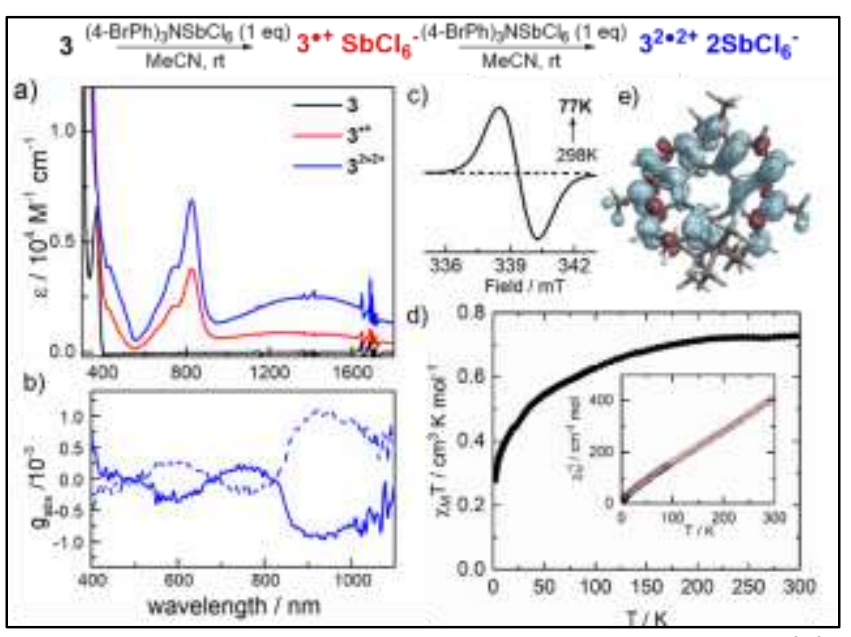

Figure 6. Synthetic pathway to form $\mathbf{3}^{\circ+} \mathbf{S b C l}_{6}^{-}$and $\mathbf{3}^{\mathbf{2 \bullet 2 +}}$ 2SbCl ${ }_{6}^{-}$; a) UV-vis-NIR absorption spectra of $\mathbf{3}$ (black), $\mathbf{3}^{\bullet+}$ $\mathbf{S b C l}_{\mathbf{6}}^{-}$(red) and $\mathbf{3}^{\mathbf{2} \mathbf{2}+} \mathbf{2 S b C l}_{\mathbf{6}}^{-}$(blue) in acetonitrile at $298 \mathrm{~K}$; b) Absorption dissymmetry factor $g_{\text {abs }}$ spectra of $(+)-\mathbf{3}^{\mathbf{2} \cdot 2+} \mathbf{2 S b C l}_{\mathbf{6}}{ }^{-}$

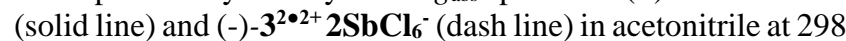
$\mathrm{K}$; c) EPR of $\mathbf{3}^{\mathbf{2} \mathbf{2}+} \mathbf{2 S b C l}_{\mathbf{6}}^{-}(\mathrm{n}=9.4858 \mathrm{GHz})$ at $77 \mathrm{~K}$ (solid line); d) $\chi_{\mathrm{M}} \mathrm{T}$ vs $\mathrm{T}$ of solid diradical $\mathbf{3}^{\mathbf{2} \mathbf{2}+} \mathbf{2} \mathbf{S b C l}_{6}{ }^{-}$; e) Calculated spin density for $(+)-3^{2 \bullet 2+}$ with an isosurface of \pm 0.002 au.

As for $\mathbf{1}^{\bullet+} \mathbf{B F}_{4}$, enantiomers of $3^{\bullet+} \mathbf{S b C l}_{6}{ }^{-}$and $\mathbf{3}^{\mathbf{2}{ }^{2+}} \mathbf{2 S b C l}_{6}{ }^{-}$remain also configurationally stable, and corresponding mirrorimage ECD spectra show similar profile up to $1100 \mathrm{~nm}$. Both $(+)-3^{\bullet+} \mathbf{S b C l}_{6}{ }^{-}$and $(+)-\mathbf{3}^{2 \bullet 2+} \mathbf{2 S b C l}_{6}{ }^{-}$displayed Cotton effects assigned to radical transitions at 660,820 and $900-950 \mathrm{~nm}(\Delta \varepsilon=$ $+0.7,-2.2$ and $+2.8 \mathrm{M}^{-1} \mathrm{~cm}^{-1}$, respectively, Figure S21), which are red-shifted in comparison to $(+)-\mathbf{1}^{\bullet+} \mathbf{B F}_{4}^{-}$, reflecting the higher electronic coupling between the carbazole $\pi$-systems. Importantly, diradical $\mathbf{3}^{\mathbf{2} \mathbf{2}+} \mathbf{2} \mathbf{S b C l}_{\mathbf{6}}^{-}$showed high chiroptical properties in the NIR region, as indicated by its absorption anisotropy factors, $\left|g_{\text {abs }}\right|=1.0 \times 10^{-3}$ at $920 \mathrm{~nm}$ (Figure 6), which is one of the highest measured for molecular organic diradicals. ${ }^{25}$, ${ }^{27}$ The magnetic properties of $\mathbf{3}^{\mathbf{2} \mathbf{2}+} \mathbf{2} \mathbf{S b C l}_{\mathbf{6}}{ }^{-}$were investigated by EPR, which resulted in an intense and broad doublet without any hyperfine coupling, nor $\Delta m=2$ half-field transition, presumably due to a weak interaction between the two radicals ( $g$ $=2.08$, Figure 6$){ }^{72}$ Given the high stability of $\mathbf{3}^{\mathbf{2} 2+} \mathbf{2 S b C l}_{\mathbf{6}}$ (more than 66 hours in solid state), magnetometry measurement were also performed on powder of $\mathbf{3}^{\mathbf{2} \boldsymbol{2}+} \mathbf{2} \mathbf{S b C l}_{6}$ at a magnetic field strength of $2 \mathrm{kOe}$ below $20 \mathrm{~K}$ and $10 \mathrm{kOe}$ above to get further insight about the interaction between radicals within the chiral environment. $\chi_{\mathrm{M}} \mathrm{T}$ is equal $0.73 \mathrm{~cm}^{3} \mathrm{~K} \mathrm{~mol}^{-1}$ at $298 \mathrm{~K}$ for $\mathbf{3}^{2 \bullet 2+} \mathbf{2 S b C l}_{6}^{-}$(Figure 6), which almost perfectly matches with the contribution for two uncoupled single electrons with $g=$ 2.00. This value started to decrease for temperatures below 170 $\mathrm{K}$, indicating an antiferromagnetic coupling between spins. $\chi_{\mathrm{M}}$ ${ }^{1}$ vs. $\mathrm{T}$ plot can be fitted with Curie-Weiss law $\left(\chi_{\mathrm{M}}=\mathrm{C} /(\mathrm{T}-\theta)\right.$ with $\mathrm{C}=0.79 \mathrm{~cm}^{3} \mathrm{~K} \mathrm{~mol}^{-1}$ and $\theta=-24 \mathrm{~K}$ (Figure 6). The Singlet-Triplet gap $\left(\Delta \mathrm{E}_{\mathrm{ST}}\right)$ estimated from $\theta$ at $67 \mathrm{~cm}^{-1}(0.19 \mathrm{kcal}$ $\mathrm{mol}^{-1}$ ) reveals a weak antiferromagnetic intramolecular spin-exchange interaction. Interestingly, these findings confirm the expected formation of a diradical upon oxidation of the highest doubly occupied molecular orbital of a monoradical with a SHI. For $\mathbf{3}^{\mathbf{2} \mathbf{2}+} \mathbf{2 S b C l}_{\mathbf{6}}^{-}$, the two unpaired electrons are localized on each carbazole fragments, with a weak overlap between the corresponding two SOMOs. ${ }^{42,44}$ Likewise, the calculations showed that $3^{2 \cdot 2+}$ has two unpaired electrons and a spin-triplet electronic state instead of a singlet configuration, including two $\alpha$-spin SOMOs and, below in energy, the set of spin-paired orbitals (Figure S64). The isosurfaces of the two SOMOs (as well as for LUMO and LUMO+1) are seen to represent in-phase and outof-phase linear combinations of frontier fragment orbitals with an energetic splitting of $0.127 \mathrm{eV}$, whose combined densities are reflected by the spin density calculations (Figure S65 and ESI for details). However, spin state energetics are not easy to deal with in DFT calculations. We found that an open-shell singlet configuration became effectively iso-energetic with the triplet when the fractions of the exact exchange in the functional went to zero, and weak interactions between the electron spins in adjacent units of $3^{\mathbf{2} \cdot 2+}$ in the solid may further complicate the picture. We thus hypothesize that, given the weak electronic coupling between the carbazole fragments in $\mathbf{3}^{\mathbf{2} \cdot \mathbf{2}} \mathbf{2} \mathbf{S b C l}_{\mathbf{6}}^{-}$, the two SOMOs could be considered as disjoint, which ultimately favored singlet ground state, in analogy with previous 3,3'-Dimethylenebiphenyl diradicals molecular design and using Ovchinnikov parity models. ${ }^{18,73,74}$

\section{CONCLUSION}

In conclusion, we described here the first persistent sterically unprotected axially chiral bicarbazole radical and its diradical analogue, along with their experimental and theoretical optical, chiroptical and magnetic properties. We investigated the relationship-structure properties of organic chiral monoradicals based on bicarbazole molecular architectures with either axial and helical chirality and found that only the former is chemically and configurationally stable, with a half-life up to 3 hours under air conditions. We attributed this unprecedented result to a complex interplay of SHI and electronic coupling between the radical center and the remaining neutral carbazole fragment, which is directly linked to the carbazoles dihedral angle and related to axial and helical chirality. Such unique electronic features provided an opportunity to design and isolate a persistent, intrinsically chiral, diradical, which displayed near infrared electronic circular dichroism intensity up to $1100 \mathrm{~nm}$ and almost degenerate singlet-triplet ground states with weak antiferromagnetic interactions evaluated by SQUID experiments $\left(\Delta \mathrm{E}_{\mathrm{ST}} \sim 0.05 \mathrm{kcal} \mathrm{mol}^{-1}\right)$. The reported findings may bring new insights on the key parameters governing the chemical and configurational stabilities of chiral organic radicals, which may offer new opportunity to design innovative organic high-spin materials.

\section{ASSOCIATED CONTENT}

Supporting Information. Methods and synthetic procedures, ORTEP diagrams, table of crystal data, unit cell diagrams, HPLC separation data, additional photophysiscal and chiroptical characterizations and theoretical calculations can be found in the Electronic Supporting Information.

\section{CORRESPONDING AUTHORS}

*jochena@buffalo.edu

*ludovic.favereau@univ-rennes1.fr

\section{NOTES}

The authors declare no competing financial interest.

\section{ACKNOWLEDGMENT}

We acknowledge the Ministère de l'Enseignement supérieur, de la Recherche et de l'Innovation, the Centre National de la Recherche Scientifique (CNRS). L. A. and J. A. thank the National Science 
Foundation (CHE-1855470) for financial support and the Center for Computational Research (CCR) at the University at Buffalo for computational resources. ${ }^{75}$ The PRISM core facility (Biogenouest $\odot$, UMS Biosit, Université de Rennes 1 - Campus de Villejean - 35043 RENNES Cedex, FRANCE) is acknowledged for the NMR characterizations and ECD measurements.

\section{REFERENCES}

1. Ardenkjær-Larsen, J. H.; Fridlund, B.; Gram, A.; Hansson, G.; Hansson, L.; Lerche, M. H.; Servin, R.; Thaning, M.; Golman, K., Increase in signal-to-noise ratio of \&gt; 10,000 times in liquid-state NMR. Proc. Natl. Acad. Sci. U. S. A. 2003, 100 (18), 10158-10163. 2. B. B. Williams; H. J. Halpern, Biomedical EPR - Part A: Free Radicals, Metals, Medicine and Physiology. Springer US: 2005.

3. Mathies, G.; Caporini, M. A.; Michaelis, V. K.; Liu, Y.; Hu, K.N.; Mance, D.; Zweier, J. L.; Rosay, M.; Baldus, M.; Griffin, R. G., Efficient Dynamic Nuclear Polarization at $800 \mathrm{MHz} / 527 \mathrm{GHz}$ with Trityl-Nitroxide Biradicals. Angew. Chem. Int. Ed. 2015, 54 (40), 11770-11774.

4. Abe, M., Diradicals. Chem. Rev. 2013, 113 (9), 7011-7088.

5. Zeng, Z.; Shi, X.; Chi, C.; López Navarrete, J. T.; Casado, J.; $\mathrm{Wu}, \mathrm{J} .$, Pro-aromatic and anti-aromatic $\pi$-conjugated molecules: an irresistible wish to be diradicals. Chem. Soc. Rev. 2015, 44 (18), 6578-6596.

6. Kumar, S.; Kumar, Y.; Keshri, S. K.; Mukhopadhyay, P., Recent Advances in Organic Radicals and Their Magnetism. Magnetochemistry 2016, 2 (4), 42.

7. Hu, X.; Wang, W.; Wang, D.; Zheng, Y., The electronic applications of stable diradicaloids: present and future. J. Mater. Chem. C 2018, 6 (42), 11232-11242.

8. Y. Gopalakrishna, T.; Zeng, W.; Lu, X.; Wu, J., From open-shell singlet diradicaloids to polyradicaloids. Chem. Commun. 2018, 54 (18), 2186-2199.

9. Kato, K.; Osuka, A., Platforms for Stable Carbon-Centered Radicals. Angew. Chem. Int. Ed. Engl. 2019, 58 (27), 8978-8986.

10. Ni, Y.; Gopalakrishna, T. Y.; Phan, H.; Kim, T.; Herng, T. S.; Han, Y.; Tao, T.; Ding, J.; Kim, D.; Wu, J., 3D global aromaticity in a fully conjugated diradicaloid cage at different oxidation states. Nat. Chem. 2020, 12 (3), 242-248.

11. Liu, C.; Ni, Y.; Lu, X.; Li, G.; Wu, J., Global Aromaticity in Macrocyclic Polyradicaloids: Huckel's Rule or Baird's Rule? Acc Chem Res 2019, 52 (8), 2309-2321.

12. Peng, Q.; Obolda, A.; Zhang, M.; Li, F., Organic LightEmitting Diodes Using a Neutral $\pi$ Radical as Emitter: The Emission from a Doublet. Angew. Chem. Int. Ed. 2015, 54 (24), 7091-7095.

13. Gu, X.; Gopalakrishna, T. Y.; Phan, H.; Ni, Y.; Herng, T. S.; Ding, J.; Wu, J., A Three-Dimensionally $\pi$-Conjugated Diradical Molecular Cage. Angew. Chem. Int. Ed. 2017, 56 (48), 1538315387.

14. Ai, X.; Chen, Y.; Feng, Y.; Li, F., A Stable Room-Temperature Luminescent Biphenylmethyl Radical. Angew. Chem. Int. Ed. 2018, 57 (11), 2869-2873.

15. Ai, X.; Evans, E. W.; Dong, S.; Gillett, A. J.; Guo, H.; Chen, Y.; Hele, T. J. H.; Friend, R. H.; Li, F., Efficient radical-based lightemitting diodes with doublet emission. Nature 2018, 563 (7732), 536-540.

16. Sugawara, T.; Matsushita, M. M., Spintronics in organic $\pi$ electronic systems. J. Mater. Chem. 2009, 19 (12), 1738-1753.

17. Sugawara, T.; Komatsu, H.; Suzuki, K., Interplay between magnetism and conductivity derived from spin-polarized donor radicals. Chem. Soc. Rev. 2011, 40 (6), 3105-3118.

18. Gallagher, N. M.; Olankitwanit, A.; Rajca, A., High-Spin Organic Molecules. J. Org. Chem. 2015, 80 (3), 1291-1298.

19. Teki, Y., Excited-State Dynamics of Non-Luminescent and Luminescent pi-Radicals. Chem. Eur. J. 2020, 26 (5), 980-996.
20. Ueda, A.; Wasa, H.; Suzuki, S.; Okada, K.; Sato, K.; Takui, T.; Morita, Y., Chiral Stable Phenalenyl Radical: Synthesis, Electronic-Spin Structure, and Optical Properties of [4]HeliceneStructured Diazaphenalenyl. Angew. Chem. Int. Ed. 2012, 51 (27), 6691-6695.

21. Wang, Y.; Zhang, H.; Pink, M.; Olankitwanit, A.; Rajca, S.; Rajca, A., Radical Cation and Neutral Radical of Azathia[7]helicene with SOMO-HOMO Energy Level Inversion. $J$. Am. Chem. Soc. 2016, 138 (23), 7298-7304.

22. Gliemann, B. D.; Petrovic, A. G.; Zolnhofer, E. M.; Dral, P. O.; Hampel, F.; Breitenbruch, G.; Schulze, P.; Raghavan, V.; Meyer, K.; Polavarapu, P. L.; Berova, N.; Kivala, M., Configurationally Stable Chiral Dithia-Bridged Hetero[4]helicene Radical Cation: Electronic Structure and Absolute Configuration. Chem. Asian J. 2017, 12 (1), 31-35.

23. Ivanov, M. V.; Thakur, K.; Bhatnagar, A.; Rathore, R., Isolation of a chiral anthracene cation radical: X-ray crystallography and computational interrogation of its racemization. Chem. Commun. 2017, 53 (18), 2748-2751.

24. Hsieh, Y. C.; Wu, C. F.; Chen, Y. T.; Fang, C. T.; Wang, C. S.; Li, C. H.; Chen, L. Y.; Cheng, M. J.; Chueh, C. C.; Chou, P. T.; Wu, Y. T., 5,14-Diaryldiindeno[2,1- f:1',2' -j]picene: A New Stable [7]Helicene with a Partial Biradical Character. J. Am. Chem. Soc. 2018, 140 (43), 14357-14366.

25. Inoue, Y.; Sakamaki, D.; Tsutsui, Y.; Gon, M.; Chujo, Y.; Seki, S., Hash-Mark-Shaped Azaacene Tetramers with Axial Chirality. J. Am. Chem. Soc. 2018, 140 (23), 7152-7158.

26. Narita, M.; Teraoka, T.; Murafuji, T.; Shiota, Y.; Yoshizawa, K.; Mori, S.; Uno, H.; Kanegawa, S.; Sato, O.; Goto, K.; Tani, F., An Azulene-Based Chiral Helicene and Its Air-Stable Cation Radical. Bull. Chem. Soc. Jpn. 2019, 92 (11), 1867-1873.

27. Shu, C.; Zhang, H.; Olankitwanit, A.; Rajca, S.; Rajca, A., High-Spin Diradical Dication of Chiral pi-Conjugated Double Helical Molecule. J. Am. Chem. Soc. 2019, 141 (43), 17287-17294. 28. Prince, R.; Tomáš, Š.; Michel, R.; Daniel, H.; Markus, N.; Martin, B.; Michal, J., Cethrene: A Helically Chiral Biradicaloid Isomer of Heptazethrene. Angew. Chem. Int. Ed. 2016, 55 (3), 1183-1186.

29. Ravat, P.; Solomek, T.; Haussinger, D.; Blacque, O.; Juricek, M., Dimethylcethrene: A Chiroptical Diradicaloid Photoswitch. $J$. Am. Chem. Soc. 2018, 140 (34), 10839-10847.

30. Shen, C.; Loas, G.; Srebro-Hooper, M.; Vanthuyne, N.; Toupet, L.; Cador, O.; Paul, F.; Lopez Navarrete, J. T.; Ramirez, F. J.; Nieto-Ortega, B.; Casado, J.; Autschbach, J.; Vallet, M.; Crassous, J., Iron Alkynyl Helicenes: Redox-Triggered Chiroptical Tuning in the IR and Near-IR Spectral Regions and Suitable for Telecommunications Applications. Angew. Chem. Int. Ed. Engl. 2016, 55 (28), 8062-6.

31. Mayorga-Burrezo, P.; Jimenez, V. G.; Blasi, D.; Parella, T.; Ratera, I.; Campana, A. G.; Veciana, J., An Enantiopure PropellerLike Trityl-Brominated Radical: Bringing Together a High Racemization Barrier and an Efficient Circularly Polarized Luminescent Magnetic Emitter. Chem. Eur. J. 2020, 26 (17), 37763781 .

32. Mayorga Burrezo, P.; Jimenez, V. G.; Blasi, D.; Ratera, I.; Campana, A. G.; Veciana, J., Organic Free Radicals as Circularly Polarized Luminescence Emitters. Angew. Chem. Int. Ed. Engl. 2019, 58 (45), 16282-16288.

33. Shil, S.; Bhattacharya, D.; Misra, A.; Klein, D. J., A high-spin organic diradical as a spin filter. Phys. Chem. Chem. Phys. 2015, 17 (36), 23378-23383.

34. Tan, J. S. J.; Paton, R. S., Frontier molecular orbital effects control the hole-catalyzed racemization of atropisomeric biaryls. Chem. Sci. 2019, 10 (8), 2285-2289.

35. Wang, W.; Wang, L.; Chen, S.; Yang, W.; Zhang, Z.; Wang, $\mathrm{X}$., Air-stable diradical dications with ferromagnetic interaction 
exceeding the thermal energy at room temperature: from a monomer to a dimer. Sci. China Chem. 2018, 61 (1674-7291), 300. 36. Gallagher, N.; Zhang, H.; Junghoefer, T.; Giangrisostomi, E.; Ovsyannikov, R.; Pink, M.; Rajca, S.; Casu, M. B.; Rajca, A., Thermally and Magnetically Robust Triplet Ground State Diradical. J. Am. Chem. Soc. 2019, 141 (11), 4764-4774.

37. Kanetomo, T.; Ichihashi, K.; Enomoto, M.; Ishida, T., Ground Triplet Spirobiradical: 2,2',7,7'-Tetra( tert-butyl)-9,9'(10 H,10' H)spirobiacridine-10,10'-dioxyl. Org. Lett. 2019, 21 (11), 3909-3912. 38. Tang, B.; Zhao, J.; Xu, J.-F.; Zhang, X., Tuning the stability of organic radicals: from covalent approaches to non-covalent approaches. Chem. Sci. 2020, 11 (5), 1192-1204.

39. There is no 'hole below the Fermi level', see ref. Gritsenko, O. V.; Baerends, E. J., The spin-unrestricted molecular Kohn-Sham solution and the analogue of Koopmans's theorem for open-shell molecules. J. Chem. Phys. 2004, 120 (18), 8364-8372. where the orbital energy of the lowest unoccupied beta spin orbital is below that of the highest occupied alpha spin orbital, and within the alpha and beta spin orbital sets there is no hole below the HOMO either. 40. Gryn'ova, G.; Marshall, D. L.; Blanksby, S. J.; Coote, M. L., Switching radical stability by $\mathrm{pH}$-induced orbital conversion. Nat. Chem. 2013, 5, 474.

41. Franchi, P.; Mezzina, E.; Lucarini, M., SOMO-HOMO Conversion in Distonic Radical Anions: An Experimental Test in Solution by EPR Radical Equilibration Technique. J. Am. Chem. Soc. 2014, 136 (4), 1250-1252.

42. Kumar, A.; Sevilla, M. D., SOMO-HOMO Level Inversion in Biologically Important Radicals. J. Phys. Chem. B 2018, 122 (1), 98-105.

43. Guo, H.; Peng, Q.; Chen, X.-K.; Gu, Q.; Dong, S.; Evans, E. W.; Gillett, A. J.; Ai, X.; Zhang, M.; Credgington, D.; Coropceanu, V.; Friend, R. H.; Brédas, J.-L.; Li, F., High stability and luminescence efficiency in donor-acceptor neutral radicals not following the Aufbau principle. Nat. Mater. 2019, 18 (9), 977-984. 44. Gryn'ova, G.; Coote, M. L.; Corminboeuf, C., Theory and practice of uncommon molecular electronic configurations. Wiley Interdisciplinary Reviews: Computational Molecular Science 2015, 5 (6), 440-459.

45. Helical chirality is often used when the considered molecule adopts a helical structure around the axis of chirality, rather than showing an $90^{\circ}$ angle arrangement between the substituents of the axis (see Mayor et al. Chem. Soc. Rev., 2016, 45, 1542). In the context of this study, we used axial and helical chirality terms to clearly point out the helical conformation adopted by $\mathbf{2}$, and the smaller dihedral angle obtained $\left(52^{\circ}\right)$ in comparison to compound $\mathbf{1}$ (angle of $87^{\circ}$ ) between the two $\pi$-conjugated carbazole systems, which directly impacts their electronic communication and the stability of their corresponding radical monocations.

46. Botman, Peter N. M.; Postma, M.; Fraanje, J.; Goubitz, K.; Schenk, H.; Maarseveen, Jan H. v.; Hiemstra, H., Synthesis and Resolution of BICOL, a Carbazole Analogue of BINOL. Eur. J. Org. Chem. 2002, 2002 (12), 1952-1955.

47. Hoen, R. New approaches in asymmetric rhodium-catalyzed hydrogenations with monodentate phosphoramidites. New approaches in asymmetric rhodium-catalyzed hydrogenations with monodentate phosphoramidites University of Groningen, 2006.

48. Gingras, M.; Felix, G.; Peresutti, R., One hundred years of helicene chemistry. Part 2: stereoselective syntheses and chiral separations of carbohelicenes. Chem. Soc. Rev. 2013, 42 (3), 10071050.

49. C.-F. Chen; Shen, Y., Helicenes Chemistry: From Synthesis to Applications. Springer, Berlin: 2017.

50. Dhbaibi, K.; Favereau, L.; Crassous, J., Enantioenriched Helicenes and Helicenoids Containing Main-Group Elements (B, Si, N, P). Chem. Rev. 2019, 119 (14), 8846-8953.

51. M. J. Frisch, G. W. T., H. B. Schlegel, G. E. Scuseria, ; M. A. Robb, J. R. C., G. Scalmani, V. Barone, ; G. A. Petersson, H. N.,
X. Li, M. Caricato, A. V. Marenich, ; J. Bloino, B. G. J., R. Gomperts, B. Mennucci, H. P. Hratchian, ; J. V. Ortiz, A. F. I., J. L. Sonnenberg, D. Williams-Young, ; F. Ding, F. L., F. Egidi, J. Goings, B. Peng, A. Petrone, ; T. Henderson, D. R., V. G. Zakrzewski, J. Gao, N. Rega, ; G. Zheng, W. L., M. Hada, M. Ehara, K. Toyota, R. Fukuda, ; J. Hasegawa, M. I., T. Nakajima, Y. Honda, O. Kitao, H. Nakai, ; T. Vreven, K. T., J. A. Montgomery, Jr., J. E. Peralta, ; F. Ogliaro, M. J. B., J. J. Heyd, E. N. Brothers, K. N. Kudin, ; V. N. Staroverov, T. A. K., R. Kobayashi, J. Normand, ; K. Raghavachari, A. P. R., J. C. Burant, S. S. Iyengar, ; J. Tomasi, M. C., J. M. Millam, M. Klene, C. Adamo, R. Cammi, ; J. W. Ochterski, R. L. M., K. Morokuma, O. Farkas, ; J. B. Foresman, D. J. F., "Gaussian 16, Revision B.01". Gaussian, Inc., Wallingford CT: 2016. URL: www.gaussian.com.

52. Adamo, C.; Barone, V., Toward reliable density functional methods without adjustable parameters: The PBE0 model. J. Chem. Phys. 1999, 110 (13), 6158-6170.

53. Weigend, F.; Ahlrichs, R., Balanced basis sets of split valence, triple zeta valence and quadruple zeta valence quality for $\mathrm{H}$ to $\mathrm{Rn}$ : Design and assessment of accuracy. Phys. Chem. Chem. Phys. 2005, 7 (18), 3297-3305.

54. Weigend, F., Accurate Coulomb-fitting basis sets for $\mathrm{H}$ to $\mathrm{Rn}$. Phys. Chem. Chem. Phys. 2006, 8 (9), 1057-1065.

55. Scalmani, G.; Frisch, M. J., Continuous surface charge polarizable continuum models of solvation. I. General formalism. J. Chem. Phys. 2010, 132 (11), 114110.

56. Grimme, S.; Antony, J.; Ehrlich, S.; Krieg, H., A consistent and accurate $a b$ initio parametrization of density functional dispersion correction (DFT-D) for the 94 elements H-Pu. J. Chem. Phys. 2010, 132 (15), 154104.

57. Autschbach, J.; Nitsch-Velasquez, L.; Rudolph, M., Timedependent density functional response theory for electronic chiroptical properties of chiral molecules. Top. Curr. Chem. 2011, 298, 1-98.

58. Srebro-Hooper, M.; Autschbach, J., Calculating Natural Optical Activity of Molecules from First Principles. Annu. Rev. Phys. Chem. 2017, 68 (1), 399-420.

59. Chiu, S. K.; Chung, Y. C.; Liou, G. S.; Su, Y. O., Electrochemical and Spectral Characterizations of 9Phenylcarbazoles. Jnl. Chinese Chemical Soc. 2012, 59 (3), 331337.

60. Karon, K.; Lapkowski, M., Carbazole electrochemistry: a short review. J. Solid State Chem. 2015, 19 (9), 2601-2610.

61. Mallick, S.; Maddala, S.; Kollimalayan, K.; Venkatakrishnan, P., Oxidative Coupling of Carbazoles: A Substituent-Governed Regioselectivity Profile. J. Org. Chem. 2019, 84 (1), 73-93.

62 . Since the term "stability" may be somehow difficult to evaluate and compare, we choose in this work to take carbazole 1' (3methoxy carbazole) as a reference for unstable radical and carbazole 3' (3-methoxy-6-tert-butylcarbazole) as a reference for stable radical (halflife of 84 hours in air) as we compare the lifetimes of carbazole-based radicals.

63. Hankache, J.; Wenger, O. S., Organic Mixed Valence. Chem. Rev. 2011, 111 (8), 5138-5178.

64. Kusamoto, T.; Kume, S.; Nishihara, H., Realization of SOMO-HOMO Level Conversion for a TEMPO-Dithiolate Ligand by Coordination to Platinum(II). J. Am. Chem. Soc. 2008, 130 (42), 13844-13845.

65. Heckmann, A.; Lambert, C., Organic Mixed-Valence Compounds: A Playground for Electrons and Holes. Angew. Chem. Int. Ed. 2012, 51 (2), 326-392.

66. Izuoka, A.; Hiraishi, M.; Abe, T.; Sugawara, T.; Sato, K.; Takui, T., Spin Alignment in Singly Oxidized Spin-Polarized Diradical Donor: Thianthrene Bis(nitronyl nitroxide). J. Am. Chem. Soc. 2000, 122 (13), 3234-3235.

67. Sakurai, H.; Izuoka, A.; Sugawara, T., Design, Preparation, and Electronic Structure of High-Spin Cation Diradicals Derived from 
Amine-Based Spin-Polarized Donors. J. Am. Chem. Soc. 2000, 122 (40), 9723-9734.

68. Nakazaki, J.; Chung, I.; Matsushita, M. M.; Sugawara, T.; Watanabe, R.; Izuoka, A.; Kawada, Y., Design and preparation of pyrrole-based spin-polarized donors. J. Mater. Chem. 2003, 13 (5), 1011-1022.

69. Komatsu, H.; Mogi, R.; Matsushita, M. M.; Miyagi, T.; Kawada, Y.; Sugawara, T., Synthesis and properties of TSF-based spin-polarized donor. Polyhedron 2009, 28 (9), 1996-2000.

70. Souto, M.; Rovira, C.; Ratera, I.; Veciana, J., TTF-PTM dyads: from switched molecular self assembly in solution to radical conductors in solid state. CrystEngComm 2017, 19 (2), 197-206.

71. Connelly, N. G.; Geiger, W. E., Chemical Redox Agents for Organometallic Chemistry. Chem. Rev. 1996, 96 (2), 877-910.

72. Eaton, S. S.; More, K. M.; Sawant, B. M.; Eaton, G. R., Use of the ESR half-field transition to determine the interspin distance and the orientation of the interspin vector in systems with two unpaired electrons. J. Am. Chem. Soc. 1983, 105 (22), 6560-6567.

73. Ovchinnikov, A. A., Multiplicity of the ground state of large alternant organic molecules with conjugated bonds. Theoretica chimica acta 1978, 47 (4), 297-304.

74. Rajca, A.; Rajca, S., Intramolecular Antiferromagnetic vs Ferromagnetic Spin Coupling through the Biphenyl Unit. J. Am. Chem. Soc. 1996, 118 (34), 8121-8126.

75. Center for Computational Research, University at Buffalo, http://hdl.handle.net/10477/79221. 
Insert Table of Contents artwork here

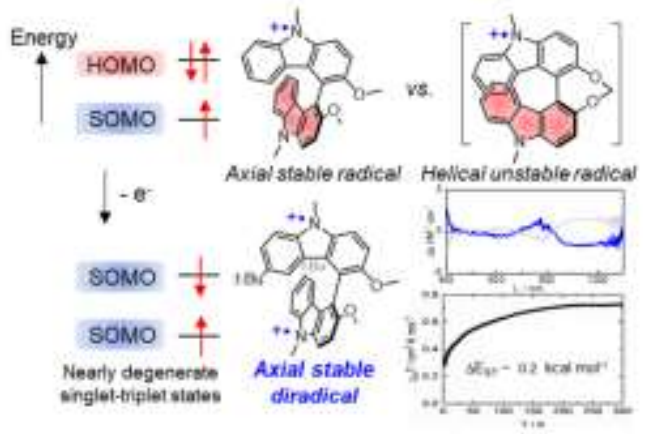

